\title{
Electron Spin-flip Scattering in Graphene due to Substrate Impurities
}

A THESIS SUBMITTED TO THE FACULTY OF THE UNIVERSITY OF MINNESOTA BY

Aditi Goswami

IN PARTIAL FULFILLMENT OF THE REQUIREMENTS FOR THE DEGREE

$\mathrm{OF}$

MASTER OF SCIENCE

P. Paul Ruden

January 2013 
(C) Aditi Goswami 2013 


\section{Acknowledgements}

Many individuals have directly or indirectly contributed towards the successful completion of this work. I would like to take this opportunity to express my sincere gratitude to each one of them. First and foremost, I want to thank my advisor, Professor P. Paul Ruden, for giving me the opportunity to work with him and for being a constant source of guidance and encouragement. I also appreciate him for taking time out for our occasional but cheerful coffee breaks. I would like to extend a special thanks to Dr. Darryl Smith, our research collaborator at Los Alamos National Laboratory for his helpful comments and suggestions. I must also thank fellow members of the Ruden group, especially Dr. Mohammad Yunus for fruitful discussions during our meetings.

My personal and professional journey at the University of Minnesota would have not come to fruition without the support of my family. I thank my parents, Kamlesh and Prakriti Ranjan Goswami for the opportunities and platforms that they have so selflessly provided for me; and my brother, Sushmit Goswami for being a role model and an inspiration. I would also like to make a special mention of Sirisha Rambhatla, for being a patient listener and a fabulous friend. Last but not the least; I want to thank Anshuman S. Nangia for his unwavering love and support over the past two years. 


\section{Dedication}

To the memory of my grandfather

Abani Kanta Goswami 


\section{Abstract}

Graphene has long been known for its peculiar Dirac-like band structure which lends it many of its remarkable properties. It is a promising material for electronic and spintronic applications due to its high carrier mobility, low intrinsic spin-orbit interaction and small hyperfine coupling. However, extrinsic effects may easily dominate intrinsic mechanisms. The scattering mechanisms investigated here are those associated with non-magnetic, charged impurities in the substrate (e.g. $\mathrm{SiO}_{2}$ ) beneath a planar n-type graphene layer. Such impurities cause an electric field that extends through the graphene and has a non-vanishing perpendicular component. Consequently, the impurity, in addition to the conventional spin-conserving scattering can give rise to spin-flip processes. The latter are a consequence of a spatially varying Rashba spin-orbit interaction caused by the electric field of the impurity in the substrate. This work focuses on the calculation of the elastic scattering cross-sections for these mechanisms. Additionally, relaxation times are estimated for assumed impurity concentrations. 


\section{Table of Contents}

List of Tables $\quad \ldots$ V

List of Figures

1. INTRODUCTION $\ldots 1$

2. THEORETICAL BACKGROUND $\ldots 4$

2.1 The Graphene Lattice $\quad \ldots 4$

2.2 Graphene Band Structure and Its Implications $\quad \ldots 6$

2.3 Spin-Orbit Coupling Effects $\quad \ldots 10$

3. POTENTIAL SCATTERING $\ldots 16$

3.1 Model Description $\quad \ldots 16$

3.2 Self-consistent Potential in the Thomas-Fermi Limit $\quad .17$

3.3 Scattering Cross-section in Born Approximation $\quad .19$

3.4 Momentum Relaxation Time $\quad \ldots 23$

4. SPIN SCATTERING $\ldots 25$

4.1 Spin-orbit Hamiltonian $\quad \ldots 25$

4.2 Scattering Cross-section $\quad \ldots 26$

4.3 Momentum and Spin Relaxation Time $\quad \ldots 30$

5. DISCUSSION AND CONCLUSION $\ldots 31$

REFERENCES $\quad \ldots 33$

iv 


\section{List of Tables}

2.1 Parameters for the calculation of spin-orbit coupling constants

$\ldots 11$

2.2 Unperturbed graphene Hamiltonian $H_{0}$ for states close to the K-point

$\ldots 12$

2.3 Spin-orbit perturbation Hamiltonian $H_{1}$ for the K-point

$\ldots 13$

4.1 Perturbation Hamiltonian $H_{1}^{\prime}$ associated with the substrate impurity

..26 


\section{List of Figures}

2.1 Lattice Structure of Graphene $\quad \ldots 5$

2.2 Reciprocal Lattice of Graphene _..5

2.3 Structure of Graphene $\pi$ and $\pi^{*}$ Bands $\quad \ldots 7$

2.4 Density of States of Graphene close to the Dirac points $\quad . .9$

$2.5 \varepsilon-k$ Plot for Graphene with Spin-orbit Coupling $\quad \ldots 14$

3.1 Modeled Device Structure $\quad \ldots 16$

3.2 Self-consistent Potential $U(\rho) \quad \ldots 19$

3.3 Elastic Scattering and Momentum Transfer $\quad \ldots 20$

3.4 Differential Scattering Cross-section for Potential Scattering _..22

3.5 Total Potential-scattering Cross-section $\quad \ldots 23$

4.1 Differential Scattering Cross-section for Spin Scattering _..28

4.2 Total Spin-scattering Cross-section $\quad \ldots 29$ 


\section{CHAPTER 1}

\section{Introduction}

Graphene is two dimensional allotrope of carbon and is often considered the building block of several other carbon allotropes such as fullerenes, carbon nanotubes and graphite. The band structure of graphene was first studied in the tight-binding approximation by Wallace in $1947 .{ }^{1}$ However, it was more than five decades later that it was first isolated from bulk graphite by micromechanical exfoliation. ${ }^{2}$

The unique electronic structure of graphene has made this material the focus of much interest for electronic device applications. ${ }^{3}$ A particularly favorable characteristic of mobile low-energy charge carriers is that elastic intravalley backscattering is effectively suppressed by the symmetry of the states near the Dirac point. Indeed, very high charge carrier mobilities have been achieved with graphene samples.

Spin transport in graphene is also a topic of growing interest for the conceivable development of spintronic devices. States of different spin mix only 
weakly in graphene due to the small intrinsic spin-orbit interaction and therefore intrinsic spin coherence times are expected to be long. ${ }^{4,5}$

It is however possible that extrinsic effects introduce a spin decoherence mechanism and impact the spin lifetimes. For example, an electric field perpendicular to the graphene plane breaks the reflection symmetry (graphene point group $\mathrm{D}_{6 \mathrm{~h}}$ ); and induces a Rashba-type spin-orbit interaction that may play a significant role in structures of the standard field effect transistor type in which charge carriers are induced into the graphene (channel) by a bias applied to a parallel conducting plate (gate). The electric field also may arise due to charged impurities in the substrate beneath the graphene layer. Effects associated with this type of field are the principal focus of this thesis. In addition, it has been shown that deviations from the perfectly planar geometry of the graphene layer (buckling) can induce relatively strong spin-orbit interaction effects. ${ }^{6}$

Chapter 2 reviews the electronic structure of graphene, and discusses the unique properties arising from the linear dispersion of low energy charge carriers. The effect of spin-orbit coupling on the band structure of graphene is also explored. In chapter 3, the modeled geometry is presented and the self-consistent potential is calculated based on a non-linear Thomas Fermi screening model. Furthermore, calculations for potential-scattering cross sections in the framework of the Born approximation are done. Rashba spin-orbit interactions are discussed 
in chapter 4 and expected spin relaxation times are calculated for an assumed substrate impurity concentration. Finally, conclusions are drawn in chapter 5 regarding the nature of the scattering mechanisms discussed and comparisons are made with other mechanisms by which spin-orbit coupling can generate spin relaxation, namely the Elliot-Yaffet and Dyakonov-Perel mechanism. 


\section{CHAPTER 2}

\section{Theoretical Background}

\subsection{The Graphene Lattice}

Graphene consists of carbon atoms arranged in a honeycomb lattice. This can be characterized as a hexagonal Bravais lattice with a two atom basis (denoted as A and B) on inequivalent sites. Each unit cell in graphene consists of two atoms, one from each sub-lattice. The nearest-neighbor atoms in graphene belong to different sub-lattices. Upon inspection it can be seen that the monolayer graphene lattice exhibits hexagonal symmetry.

The corresponding reciprocal lattice is also hexagonal, and it is at the corners of the hexagonal Brillouin zone (BZ) that the conduction and valence bands originating from the $\pi$ and $\pi^{*}$ bands touch. The six corners of the BZ are often referred to as the Dirac points or the K and K' points in order to differentiate between the inequivalent $\mathrm{BZ}$ corners. 


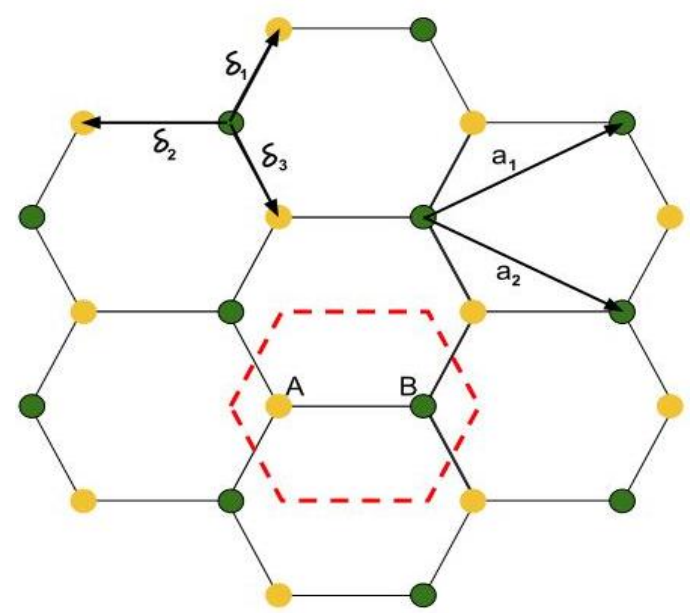

Figure 2.1: Lattice structure of graphene: $a_{1}$ and $a_{2}$ are the lattice unit vectors while $\delta_{1}, \delta_{2}$ and $\delta_{3}$ are the nearest-neighbor vectors. The primitive unit cell of graphene with a two atom basis is shown with dashed lines.

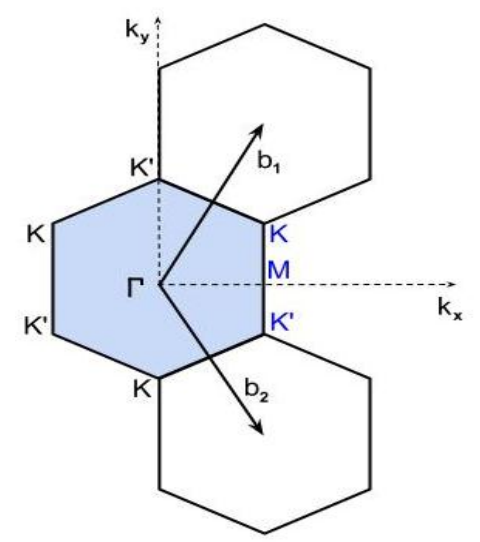

Figure 2.2: Reciprocal lattice of graphene: $b_{1}$ and $b_{2}$ are the reciprocal lattice vectors while the $\Gamma$-point is the center of the BZ. Coordinates of the highlighted points with respect to the zone center are $M=(2 \pi / \sqrt{ } 3 a, 0), K=(2 \pi / \sqrt{ } 3 a, 2 \pi / 3 a)$ and $K^{\prime}=(2 \pi / \sqrt{ } 3 a,-2 \pi / 3 a)$ where $a$ is the lattice constant and equal to $2.46 \AA \hat{~}$ 


\subsection{Graphene Band Structure and its Implications}

Following the tight-binding approach presented in Ref. (7), the Schrodinger equation for the $\pi$ and $\pi^{*}$ states of graphene can be written as:

$$
\left[\begin{array}{cc}
0 & h_{A B}(\vec{\kappa}) \\
h_{A B}{ }^{*}(\vec{\kappa}) & 0
\end{array}\right]\left[\begin{array}{l}
f_{A}(\vec{\kappa}) \\
f_{B}(\vec{\kappa})
\end{array}\right]=\varepsilon_{\kappa}\left[\begin{array}{l}
f_{A}(\vec{\kappa}) \\
f_{B}(\vec{\kappa})
\end{array}\right]
$$

Here, $\quad h_{A B}(\vec{\kappa})=-\gamma_{0} \sum_{l} \exp \left(-i \vec{\kappa} \cdot \overrightarrow{\delta_{l}}\right)$ with $-\gamma_{0}$ and $\overrightarrow{\delta_{l}}$ being the transfer integral between the nearest neighbor carbon atoms and the vectors connecting nearest neighbor carbon atoms, respectively. To describe states in the vicinity of the $\mathrm{K}$ and $\mathrm{K}$ ' points, we use the k.p method based on the above matrix and introduce a new vector $\vec{k}=\vec{\kappa}-\overrightarrow{\mathrm{K}}$ with $\vec{k}<<|\vec{\kappa}|$.

Expanding $h_{A B}(\vec{\kappa})$ and using the symmetry of atoms that are $120^{\circ}$ apart, we get the following expression:

$\hbar v_{f}\left[\begin{array}{cc}0 & \left.\left(k_{x}-i k_{y}\right)\right) \\ \left(k_{x}+i k_{y}\right) & 0\end{array}\right]\left[\begin{array}{l}f_{1}(\vec{k}) \\ f_{2}(k)\end{array}\right]=\varepsilon_{\kappa}\left[\begin{array}{l}f_{1}(\vec{k}) \\ f_{2}(\vec{k})\end{array}\right]$

In the above expression, $v_{f}$ is the Fermi velocity and is given by:

$v_{f}=\frac{\sqrt{3}}{2 \hbar} a \gamma_{0}$ 


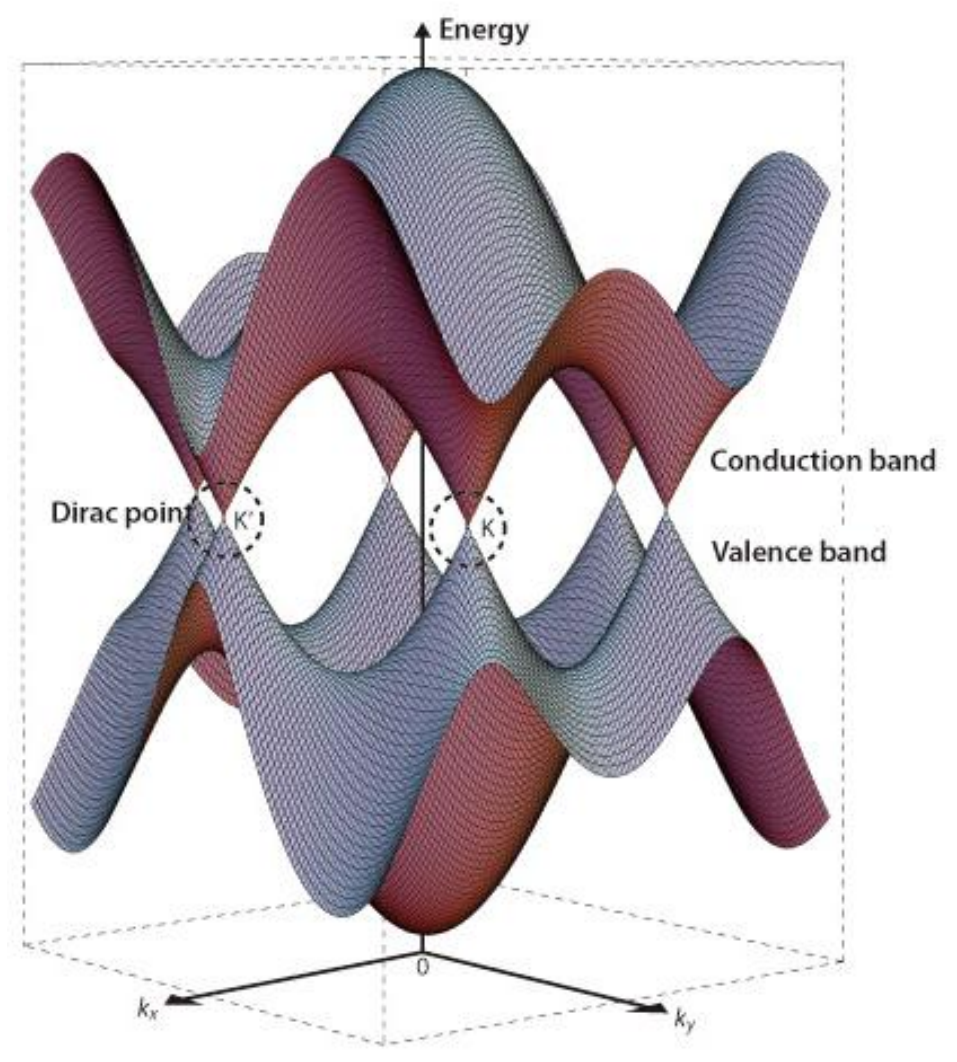

Figure 2.3: ${ }^{8}$ Structure of graphene $\pi$ and $\pi^{*}$ bands over the complete Brillouin zone

Diagonalizing the $2 \times 2$ Hamiltonian yields a linear dispersion relationship:

$\varepsilon_{k}= \pm \hbar v_{f}|\vec{k}|$

('+' and '-' represent conduction and valence band states, respectively) 
The linear dispersion deduced above can be used to calculate the 2-D density of states for a unit cell of area $\Omega$ for states close to the Dirac point or the zero- energy ${ }^{9}$ :

$\tilde{g}(k) d k=2 \times 2 \frac{2 \pi k d k}{(2 \pi)^{2} / \Omega}$

In the above expression, the spin and valley degeneracy (which arises from the fact that each of the six Dirac points are shared by three hexagons) each contribute a pre-factor of two. The density of states (per unit area) is therefore given by:

$$
g(E)=\frac{2}{\pi}\left|k\left(\frac{d E}{d k}\right)^{-1}\right|=\frac{2|E|}{\pi \hbar^{2} v_{f}^{2}}
$$

Hence, close to the Dirac point the density of states is linear in the energy, $E$ and at $E=0$, the density of states (DOS) vanishes.

Other striking conclusions can be drawn from the linear $\varepsilon-k$ dispersion in graphene. First, the effective mass is zero at zero energy due to infinite curvature at the Dirac point and, second, the Fermi velocity in equation (2.3) depends neither on energy nor on momentum. 


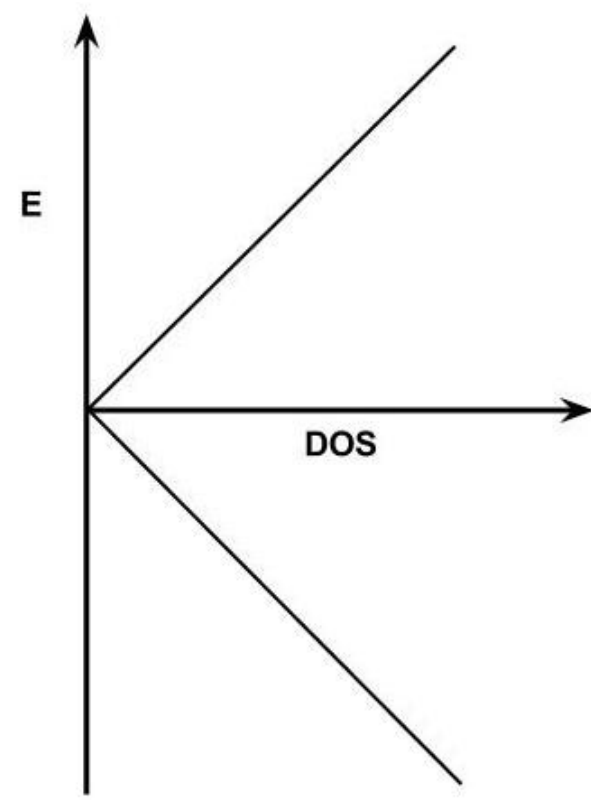

Figure 2.4: Density of states of graphene close to the Dirac points

For $\varepsilon_{k}>0$, the eigenfunctions at the $\mathrm{K}$ and $\mathrm{K}$ ' point are respectively given by ${ }^{10}$ :

$\left[\begin{array}{l}f_{1}(\vec{k}) \\ f_{2}(\vec{k})\end{array}\right]=\frac{1}{\sqrt{2}}\left[\begin{array}{c}e^{-i \theta_{k} / 2} \\ e^{i \theta_{k} / 2}\end{array}\right]$ and $\left[\begin{array}{l}f_{1}(\vec{k}) \\ f_{2}(\vec{k})\end{array}\right]=\frac{1}{\sqrt{2}}\left[\begin{array}{c}e^{i \theta_{k} / 2} \\ e^{-i \theta_{k} / 2}\end{array}\right]$

where $\theta_{k}=\arctan \left(\frac{k_{x}}{k_{y}}\right)$

Upon inspection of figure 2.2 and the above formulation, it can be seen that the eigenfunctions at the Dirac point are mirror-symmetric along the $k_{x}$-axis i.e. $\left(k_{x}, k_{y}\right) \Leftrightarrow\left(k_{x},-k_{y}\right)$. Moreover, if the phase $\theta_{k}$ is rotated by $2 \pi$ then the eigenfunctions change sign i.e. the wavefunctions acquire a "Berry's phase". 
Another unique property and a manifestation of the chiral graphene wavefunctions is the suppression of backscattering. The eigenfunctions of the Kpoint are given by equation (2.7) as:

$\left[\begin{array}{l}f_{1}(\vec{k}) \\ f_{2}(\vec{k})\end{array}\right]=\frac{1}{\sqrt{2}}\left[\begin{array}{c}e^{-i \theta_{k} / 2} \\ e^{i \theta_{k} / 2}\end{array}\right]$

An incoming $\vec{k}$ state would backscatter as an outgoing $-\vec{k}$ state i.e. $\theta_{-k}=\theta_{k}+\pi$

$\Rightarrow\left[\begin{array}{l}f_{1}(-\vec{k}) \\ f_{2}(-\vec{k})\end{array}\right]=\frac{-i}{\sqrt{2}}\left[\begin{array}{l}e^{-i \theta_{k} / 2} \\ -e^{i \theta_{k} / 2}\end{array}\right]$

As can be seen from equations (2.7) and (2.8), $\left[\begin{array}{ll}f_{1}{ }^{*}(\vec{k}) & f_{2}{ }^{*}(\vec{k})\end{array}\right]\left[\begin{array}{l}f_{1}(-\vec{k}) \\ f_{2}(-\vec{k})\end{array}\right]=0$

i.e. states with opposite $\vec{k}$ are orthogonal and consequently backscattering is suppressed in graphene.

\subsection{Spin-Orbit Coupling Effects}

The picture presented in the previous sections breaks down when relativistic effects such as spin-orbit interactions are included in the band dispersion calculation. If the mirror symmetry about the plane is preserved, then the only spin dependent term needed is due to the intrinsic spin-orbit coupling i.e. the Dresselhaus term, $\Delta_{\mathrm{so}}$. However, in the presence of a symmetry-breaking field (e.g. a perpendicular electric field $F$ ), the Rashba term $(R)$ is also required. For 
these two cases, the respective coupling constants are given by the following expressions:

$$
\begin{aligned}
& \Delta_{s o}=\frac{|s|}{18(s p \sigma)^{2}} \xi^{2} \\
& R=\frac{e F b_{0}}{3(s p \sigma)} \xi
\end{aligned}
$$

In the above expressions, $\xi$ is the strength of the spin-orbit interaction, $|s|$ and $(s p \sigma)$ are tight-binding model parameters, and $\mathrm{b}_{0}$ is a length comparable to the size of the carbon atom. Clearly, the numerical value of the Rashba term depends linearly on the electric field perpendicular to the graphene plane.

Table 2.1: Parameters for the calculation of the spin-orbit coupling constants ${ }^{6,11,12}$

\begin{tabular}{|c|c|}
\hline$b_{0}$ & $1.86 \times 10^{-8} \mathrm{~cm}$ \\
\hline$\xi$ & $6.0 \mathrm{meV}$ \\
\hline$s p \sigma$ & $5.58 \mathrm{eV}$ \\
\hline$|s|$ & $|-8.868| \mathrm{eV}$ \\
\hline
\end{tabular}


The above parameters yield $\Delta_{S O}=5.7 \times 10^{-4} \mathrm{meV}$ and $R=0.0111 \mathrm{meV}$ (for a uniform field strength of $F=50 \mathrm{~V} / 300 \mathrm{~nm}$ ) for the Dresselhaus and Rashba coupling constants, respectively.

In the effective mass approximation, the Hamiltonian in the vicinity of the Dirac points can be expressed as an $8 \times 8$ matrix. Assuming no coupling between the $\mathrm{K}$ and $\mathrm{K}$ ' points, we can reduce the $8 \times 8$ Hamiltonian into two $4 \times 4$ matrices. Using the standard sub-lattice representation of spin eigenfunctions, the unperturbed graphene Hamiltonian, $\mathrm{H}_{0}$, for states of both spin directions close to the K-point is shown by table 2.2:

Table 2.2: Unperturbed graphene Hamiltonian $\mathrm{H}_{0}$ for states close to the K-point
$\mathrm{A}, \uparrow$
$\mathrm{B}, \uparrow$
$\mathrm{A}, \downarrow$
$\mathrm{B}, \downarrow$

\begin{tabular}{c|c|c|c|c|}
$\mathrm{A}, \uparrow$ & 0 & $\hbar v_{f}\left(k_{x}+i k_{y}\right)$ & 0 & 0 \\
\cline { 2 - 5 } $\mathrm{B}, \uparrow$ & $\hbar v_{f}\left(k_{x}-i k_{y}\right)$ & 0 & 0 & 0 \\
\cline { 2 - 5 },$\downarrow$ & 0 & 0 & 0 & $\hbar v_{f}\left(k_{x}+i k_{y}\right)$ \\
\cline { 2 - 5 } & 0 & 0 & $\hbar v_{f}\left(k_{x}-i k_{y}\right)$ & 0 \\
\hline
\end{tabular}

Setting the zero-energy to be at the bottom of the conduction band, the spin-orbit perturbation Hamiltonian $\left(\mathrm{H}_{1}\right)$ for the K-point has the form given by table 2.3: ${ }^{11}$ 
Table 2.3: Spin-orbit perturbation Hamiltonian $\mathrm{H}_{1}$ for the $\mathrm{K}$-point
A, $\uparrow$
$\mathrm{B}, \uparrow$
A, $\downarrow$
$\mathrm{B}, \downarrow$

\begin{tabular}{c|c|c|c|c|}
$\mathrm{A}, \uparrow$ & 0 & 0 & 0 & 0 \\
\cline { 2 - 5 } $\mathrm{B}, \uparrow$ & 0 & $-2 \Delta_{s o}$ & $-2 i R$ & 0 \\
\cline { 2 - 5 } $\mathrm{A}, \downarrow$ & $2 i R$ & $-2 \Delta_{s o}$ & 0 \\
\cline { 2 - 5 } & 0 & 0 & 0 & 0 \\
\hline
\end{tabular}

When the mirror symmetry is preserved, i.e. $F=0$ (and consequently $R=0$ ) then the following energy eigenvalues are obtained for the K-point, with each of the energy eigenvalues being two-fold degenerate:

$\varepsilon_{k}=-\Delta_{S O} \pm \sqrt{\Delta_{S O}^{2}+\hbar^{2} v_{f}^{2} k^{2}}$ where $k^{2}=k_{x}^{2}+k_{y}^{2}$

With the Dresselhaus term alone, an energy gap of $2 \Delta_{S O}$ is introduced at the Dirac point. However if we account for the presence of an electric field $F$ and introduce the Rashba term, the following eigenvalues are obtained by diagonalizing the $4 \times 4$ K-point Hamiltonian:

$\varepsilon_{k, c}= \pm R-\Delta_{S O}+\sqrt{\left(R \mp \Delta_{S O}\right)^{2}+\hbar^{2} v_{f}^{2} k^{2}}$ (conduction band energies)

$\varepsilon_{k, v}=\mp R-\Delta_{S O}-\sqrt{\left(\Delta_{S O} \pm R\right)^{2}+\hbar^{2} v_{f}^{2} k^{2}}$ (valence band energies) 


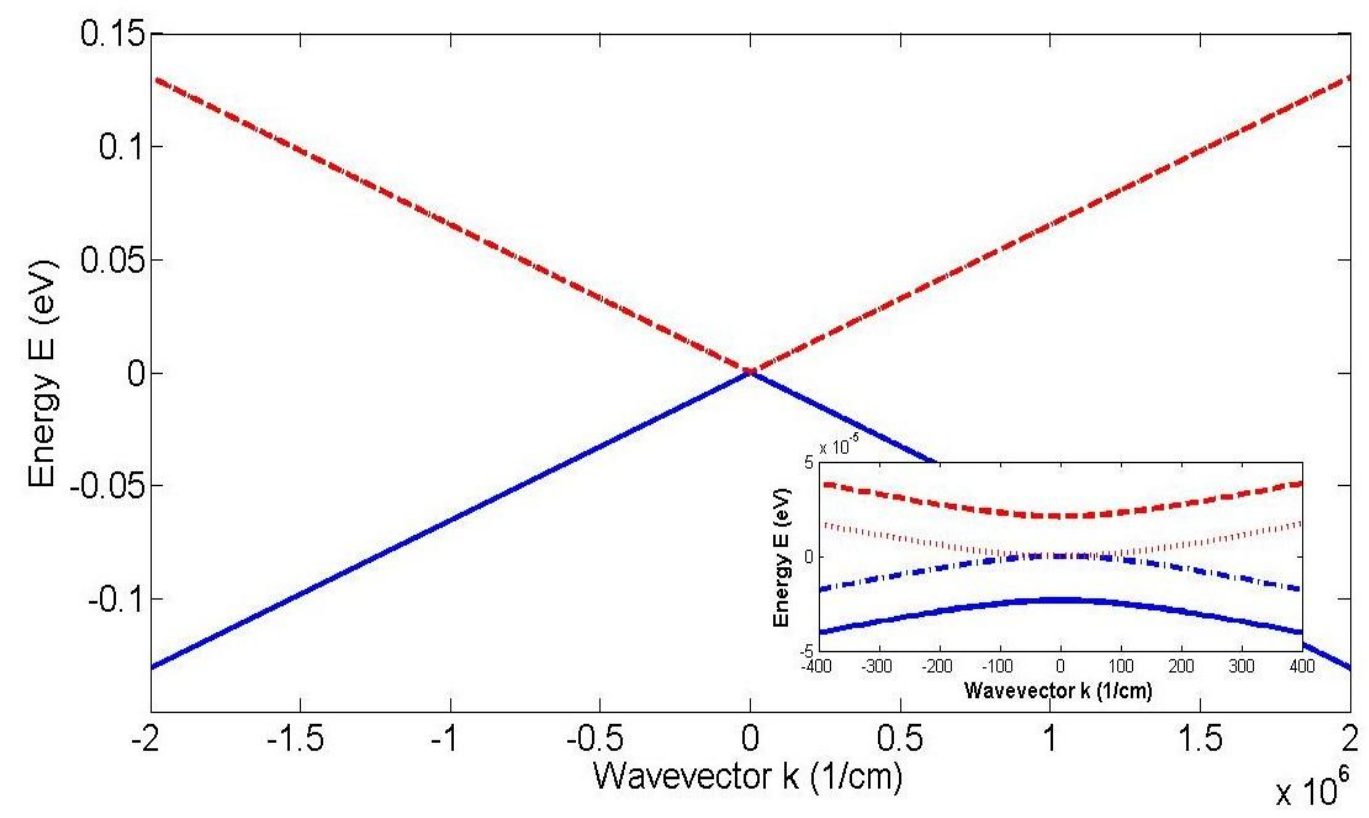

Figure 2.5: \&-k plot for graphene when spin-orbit coupling is taken into account

$$
\text { (for }|R|>\Delta_{\text {so }} \text {, no band gap is observed) }
$$

As can be seen, the two-fold degeneracy is lifted by the Rashba effect and the energy band gap at the K-point is $2\left|\Delta_{S O}-R\right|$ when $|R|<\Delta_{S O}$. However, numerical estimates for the two terms suggest that $\Delta_{S O}<|R|$ in many experimentally realized cases, a condition under which the energy gap closes and the bands disperse quadratically near the Dirac point. 
Band structure calculations for graphene that do not take into account relativistic effects yield a linear energy dispersion relationship with no band gap. Spin dependent terms however, can give rise to qualitative changes in the energy band structure of graphene, the extent of which depends on their magnitudes relative to one another. 


\section{CHAPTER 3}

\section{Potential Scattering}

\subsection{Model Description}

This chapter presents a model to describe the effect a charged substrate impurity on the elastic scattering of charge carriers in graphene. The device structure envisioned for the model is shown in figure 3.1. An atomically thin and infinite sheet of n-type graphene rests on a $\mathrm{SiO}_{2}$ substrate layer with a single, ionized impurity a distance $\mathrm{z}_{0}$ below the graphene- $\mathrm{SiO}_{2}$ interface. The modelled geometry is cylindrically symmetric.

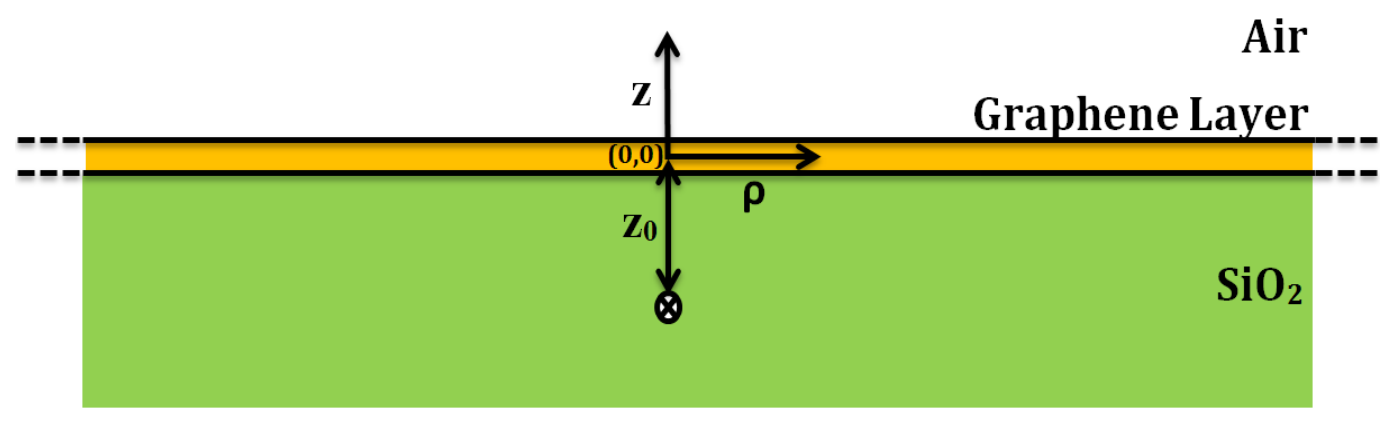

Figure 3.1: Model Device Structure used in the present work: a single impurity atom with charge $+e$ is at distance $\mathrm{z}_{0}$ below the graphene-SiO $\mathrm{S}_{2}$ interface 


\subsection{Self-Consistent Potential in the Thomas-Fermi Limit}

The potential acting on the electrons at $\mathrm{z}=0$ (graphene plane) due to the impurity charge $+e$ situated at $\mathrm{z}_{0}(<0)$ is: ${ }^{13}$

$U_{0}(\vec{\rho})=-\frac{2 e^{2}}{1+\kappa} \frac{1}{\sqrt{\vec{\rho}^{2}+z_{0}^{2}}}$

where $\kappa$ is the dielectric constant of $\mathrm{SiO}_{2}$.

Hence, the charged impurity in the substrate gives rise to an electric field in its vicinity, resulting in a spatially varying electron distribution in the graphene. Since the graphene DOS varies strongly with energy near the Dirac point, a nonlinear Thomas-Fermi description of screening is employed. At $\mathrm{T}=0$, the induced carrier density in graphene is then given by:

$n_{S}(\vec{\rho})=\int_{\varepsilon_{F}}^{\varepsilon_{F}-U(\vec{\rho})} g(E) d E$

(where $g(E)$ is the density of states for graphene, given by equation 2.4)

The induced potential due to the sheet charge is given by:

$U_{\text {ind }}(\vec{\rho})=\frac{2 e^{2}}{1+\kappa} \int d^{2} \rho^{\prime} n_{s}\left(\overrightarrow{\rho^{\prime}}\right) \frac{1}{\left|\vec{\rho}-\overrightarrow{\rho^{\prime}}\right|}$

Taking into consideration that the induced density and the potentials have cylindrical symmetry, this expression simplifies to: 
$\Rightarrow U_{\text {ind }}(\rho)=\frac{2 e^{2}}{1+\kappa} \int_{0}^{\infty} \rho^{\prime} d \rho^{\prime} n_{s}\left(\rho^{\prime}\right) \frac{4}{\left(\rho+\rho^{\prime}\right)} K\left(\frac{4 \rho \rho^{\prime}}{\left(\rho+\rho^{\prime}\right)^{2}}\right)$

(where $\mathrm{K}$ is the complete elliptic integral of the first kind)

The overall potential due the charged impurity is therefore, $U(\rho)=$ $U_{\text {ind }}(\rho)+U_{0}(\rho) . U(\rho)$ is solved for in Fourier space in an iterative fashion until the potential and charge density are self-consistent. Figure 3.2 plots the selfconsistent potential as a function of $\rho$ for three different electron densities corresponding to Fermi energies $\varepsilon_{\mathrm{f}} \sim 10 \mathrm{meV}, 50 \mathrm{meV}$ and $100 \mathrm{meV}$. 


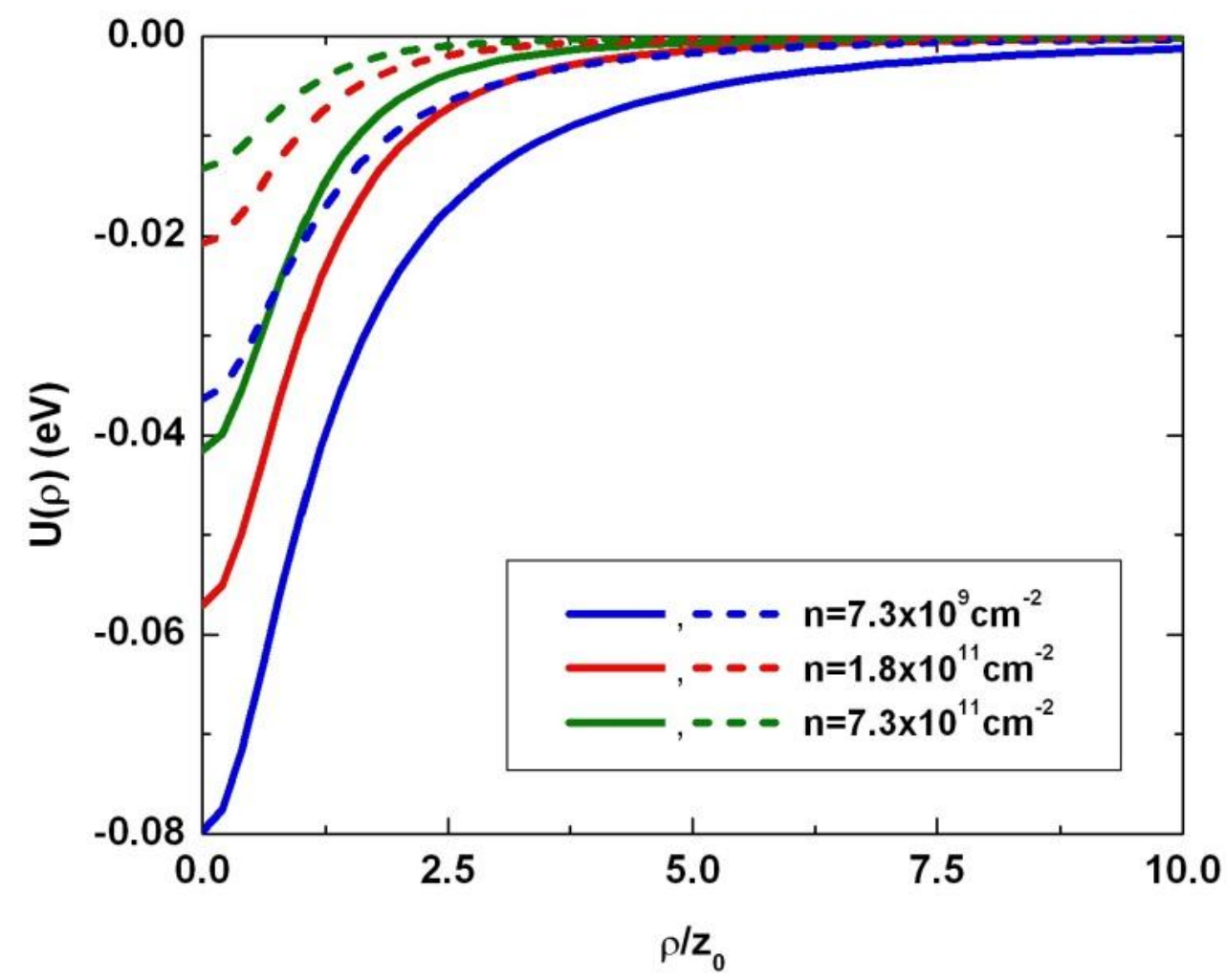

Figure 3.2: Self-consistent potential $U(\rho)$ as a function of $\rho$ for several different bulk electron densities. Distances of $z_{0}=5 \mathrm{~nm}$ (dashed lines) and $2.5 \mathrm{~nm}$ (solid lines) are assumed.

\subsection{Scattering Cross-section in Born Approximation}

Carriers in the graphene monolayer can undergo elastic scattering from the

cylindrically symmetric potential induced by the charged substrate impurity. If $\vec{k}$ is the incoming state and $\vec{k}^{\prime}$ is the outgoing state, then the momentum transfer is 
$\vec{q}=\vec{k}^{\prime}-\vec{k}$. Since the scattering is elastic $\left|\overrightarrow{k^{\prime}}\right|=|\vec{k}|$ and for a scattering angle of $\theta$; the momentum transfer $q=2 k \sin (\theta / 2)$

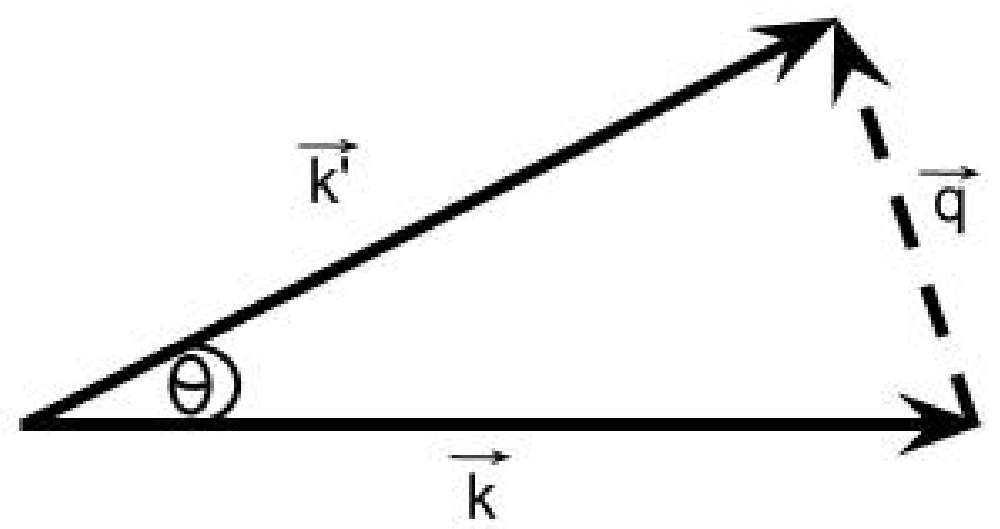

Figure 3.3: Elastic scattering and momentum transfer $\left(\vec{k}\right.$ and $\overrightarrow{\boldsymbol{k}^{\prime}}$ are the incident and scattered wave while $\vec{q}$ is the momentum transfer)

The potential scattering differential and total cross sections are respectively given by:

$$
\begin{aligned}
& \frac{d \sigma_{P}}{d \theta}=|f(\theta)|^{2} \\
& \sigma_{P}=\oint|f(\theta)|^{2} d \theta
\end{aligned}
$$

where $f(\theta)$ is the 2-D scattering amplitude. 
In the Born approximation, the scattering amplitude is: ${ }^{14}$

$f_{b o r n}(\theta)=-\frac{1}{\hbar v_{f}} \sqrt{\frac{k}{8 \pi}} U(q)\left(1+e^{-i \theta}\right)$

$U(q)$ is the marix element that couples the incoming and outgoing state and is the 2-D Fourier transform of the potential $U(\rho)$ i.e. $U(q)=\int d^{2} \rho e^{-i \vec{q} \cdot \vec{\rho}} U(\rho)$. Because of the cylindrical symmetry of the potential:

$U(\vec{\rho})=U(\rho)$ and $U(\vec{q})=U(q)$

Therefore in the Born approximation, the differential cross-section is:

$\Rightarrow \frac{d \sigma_{P}}{d \theta}=\frac{4}{\hbar^{2} v_{f}^{2}}\left(\frac{k}{8 \pi}\right)|U(q)|^{2} \cos ^{2}\left(\frac{\theta}{2}\right)$

Figure 3.4 plots the differential cross-section for potential scattering as a function of the scattering angle for three different electron energies, and $n=7.3 \times$ $10^{9} \mathrm{~cm}^{-2}, 1.8 \times 10^{11} \mathrm{~cm}^{-2}$ and $7.3 \times 10^{11} \mathrm{~cm}^{-2}$. Evidently, backscattering (as discussed in section 2.2) is suppressed by the last term in the above equation. 


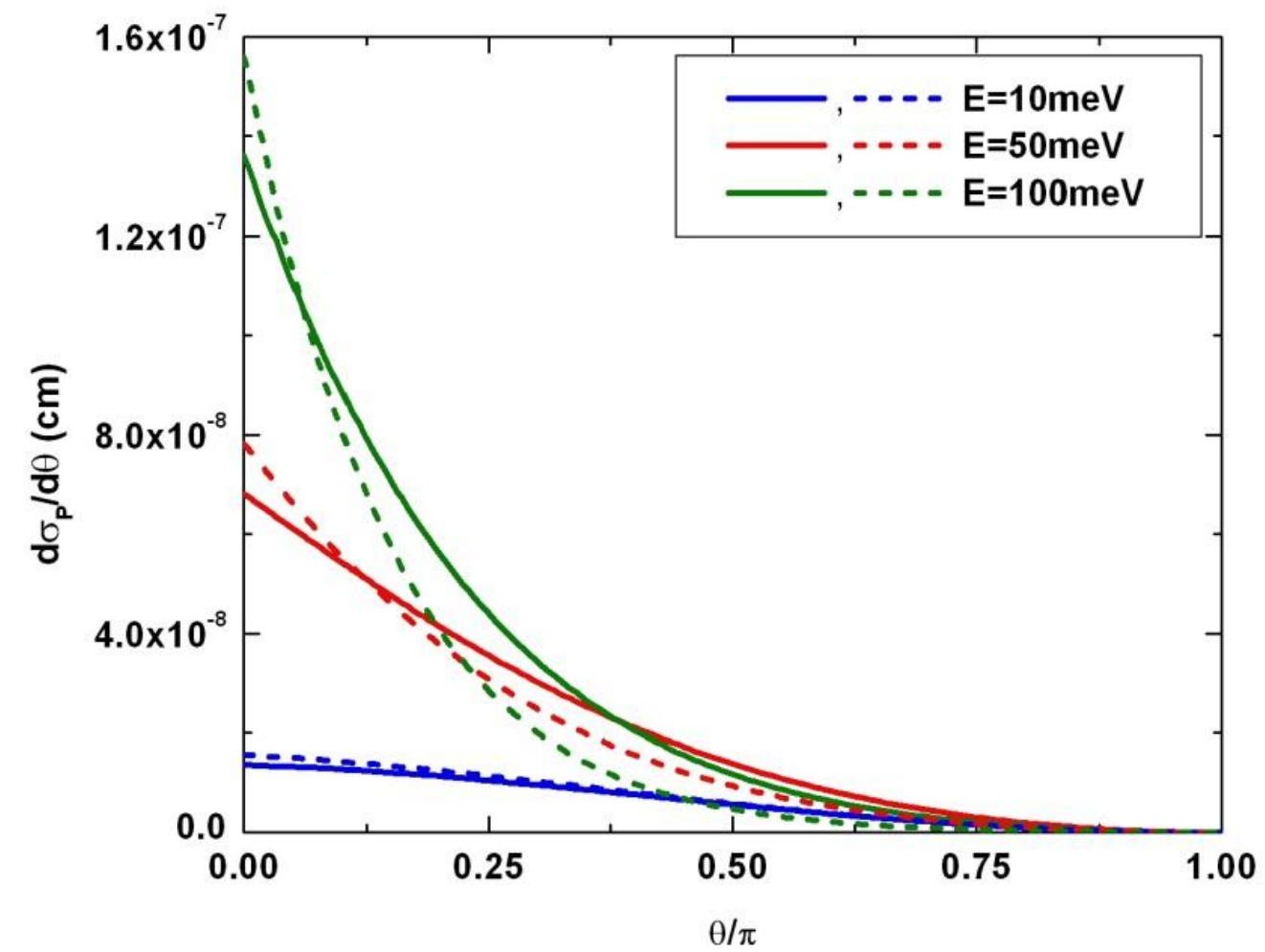

Figure 3.4: Differential scattering cross-section for potential scattering as a function of $\theta$ for several different electron energies, $z_{0}=5 \mathrm{~nm}$ (dashed lines) and $2.5 \mathrm{~nm}$ (solid lines).

The differential cross-section is integrated over the scattering angle to obtain the total potential-scattering cross-section. Figure 3.5 plots this total crosssection for potential scattering as a function of electron energy. As interest focuses primarily on electrons near the Fermi energy, the energy scale is normalized. 


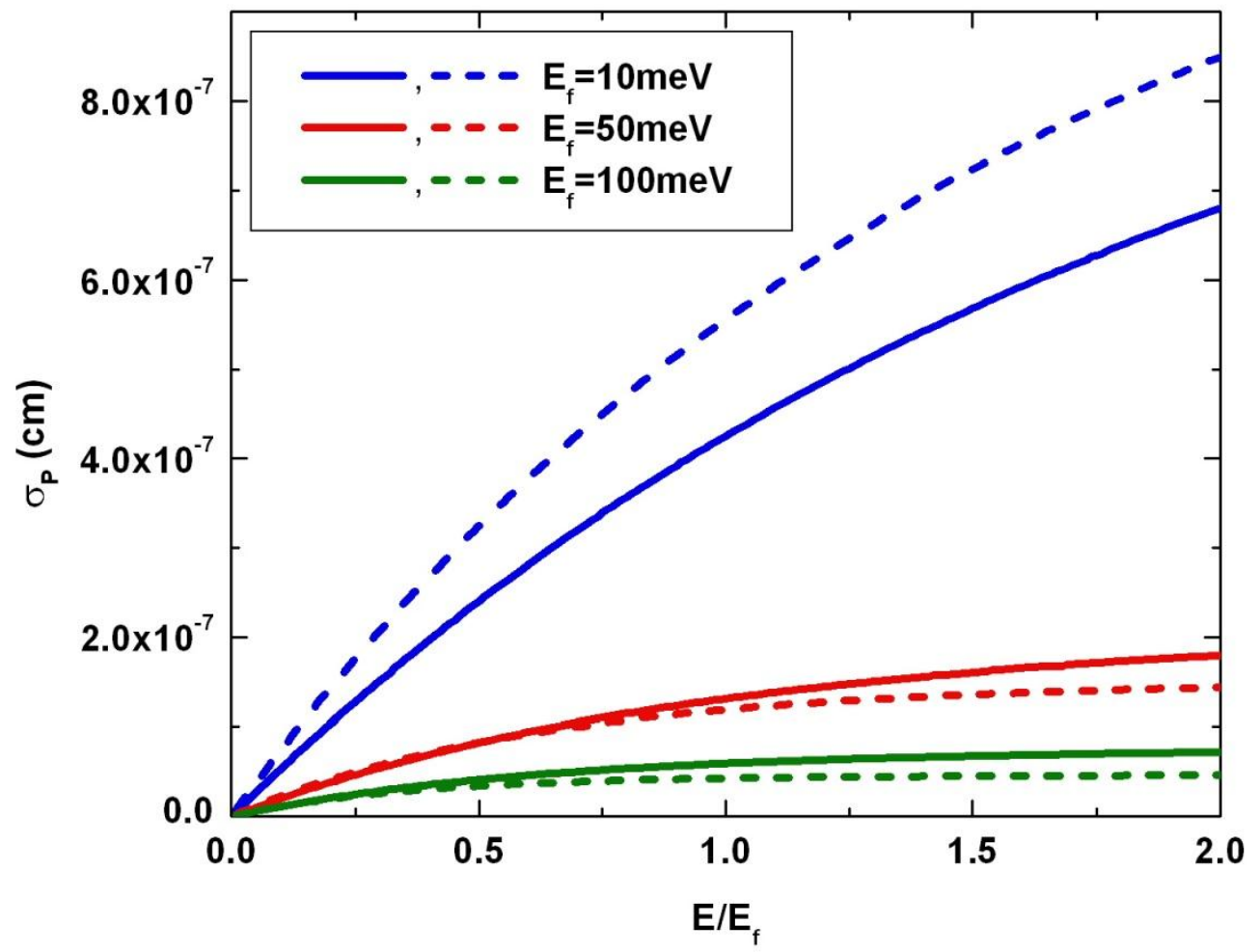

Figure 3.5: Total potential-scattering cross-section as a function of electron energy, $z_{0}=5 \mathrm{~nm}$ (dashed lines) and 2.5nm (solid lines).

\subsection{Momentum Relaxation Time}

Potential scattering can contribute to momentum relaxation. However, small angle scattering i.e. $\theta \approx 0$, does not contribute much to momentum relaxation. In 
order to take this into account, a factor of $(1-\cos \theta)$ is included in the calculation of the differential momentum cross-section, and, since

$$
\begin{aligned}
& \cos ^{2}\left(\frac{\theta}{2}\right)=\left(\frac{1}{2}\right)(1+\cos \theta): \\
& \frac{d \sigma_{m, P}}{d \theta}=\frac{1}{\hbar^{2} v_{f}^{2}}\left(\frac{k(\epsilon)}{4 \pi}\right)|U(\vec{q})|^{2} \sin ^{2}(\theta)
\end{aligned}
$$

where $\theta$ is the scattering angle and $\epsilon$ is the energy of the incoming and outgoing states.

The momentum relaxation time can then be defined as:

$$
\tau_{m, P}^{-1}(\epsilon)=v_{f} N_{i}^{(2)} \sigma_{m, P}(\epsilon)
$$

Here, $N_{i}{ }^{(2)}$ is a 2-D density of the substrate impurities while $\sigma_{m . P}(\epsilon)$ is the total momentum scattering cross-section obtained by integrating the differential momentum cross-section over $\theta$. Assuming the impurity density to be $10^{11} \mathrm{~cm}^{-2}$, $z_{0}=2.5 \mathrm{~nm}$ and for electrons at the Fermi energy $\left(E_{\mathrm{f}}=50 \mathrm{meV}\right)$ the potential scattering momentum relaxation time is $\tau_{\mathrm{m}, \mathrm{p}} \approx 2 p s$. 


\section{CHAPTER 4}

\section{Spin Scattering}

\subsection{Spin-Orbit Hamiltonian}

Considering that the graphene layer in the previously described model is doped, two types of impurities can be envisioned. For impurities that lie in-plane, the field perpendicular to the graphene surface vanishes. However, out-of-plane impurities like the one envisioned in figure 3.1 have a non-zero electric field component in the z-direction. This causes a spatially varying electric field perpendicular to the graphene and, hence, a spatially varying Rashba spin-orbit interaction results. The perpendicular electric field can induce (elastic) transitions between states of opposite spin via the local Rashba interaction, which may or may not be associated with crystal momentum relaxation.

If we neglect the small intrinsic spin-orbit interaction, then the perturbation Hamiltonian $\left(H_{1}^{\prime}\right)$ associated with the substrate impurity is given by table 4.1: 
Table 4.1: Perturbation Hamiltonian $H_{1}^{\prime}$ associated with the substrate impurity
$\mathrm{A}, \uparrow$
$\mathrm{B}, \uparrow$
$\mathrm{A}, \downarrow$
$\mathrm{B}, \downarrow$

\begin{tabular}{c|c|c|c|c|}
$\mathrm{A}, \uparrow$ & $U(\rho)$ & 0 & 0 & 0 \\
\cline { 2 - 5 } $\mathrm{B}, \uparrow$ & 0 & $U(\rho)$ & $-2 i R(\rho)$ & 0 \\
$\mathrm{~A}, \downarrow$ & $2 i R(\rho)$ & $U(\rho)$ & 0 \\
\cline { 2 - 5 } $\mathrm{B}, \downarrow$ & 0 & 0 & 0 & $U(\rho)$ \\
\hline
\end{tabular}

Here $U(\rho)$ is the screened impurity potential while $R(\rho)$ is the Rashba term first introduced in Chapter 2. The latter depends on the perpendicular electric-field which is calculated analytically to be:

$e F(\rho)=-\frac{2 e^{2}}{1+\kappa}\left[\frac{z_{0}}{\left(\rho^{2}+z_{0}^{2}\right)^{3 / 2}}\right]$

(In this model the combined potential due to the charge in the graphene and the polarization charge at the $\mathrm{SiO}_{2}$-graphene interface is symmetric in $z$ and the average perpendicular field across the graphene is therefore taken to be zero.)

\subsection{Scattering Cross-section}

In the lowest order Born approximation, the differential cross-section for spin scattering due to the Rashba term in the above Hamiltonian is given by: 
$\frac{d \sigma_{R}}{d \theta}=\frac{1}{4 \hbar^{2} v_{f}^{2}}\left(\frac{k}{8 \pi}\right)|R(q)|^{2}$

In the above expression, $R(q)$ is the Rashba term in Fourier space and is defined as:

$R(q)=\frac{b_{0} \xi}{3(s p \sigma)} e F(q)$

(where $F(q)$ is the 2-D Fourier transform of the perpendicular electric field $F(\rho)$ given by equation 4.1 )

Unlike potential scattering, backscattering is not suppressed in this case as the initial and final states have opposite spin. Results for the differential crosssection associated with the Rashba interaction due to the impurity field are plotted as a function of the scattering angle in figure 4.1, again for three different electron energies corresponding to the Fermi energies for $n=7.3 \times 10^{9} \mathrm{~cm}^{-2}, 1.8 \times$ $10^{11} \mathrm{~cm}^{-2}$ and $7.3 \times 10^{11} \mathrm{~cm}^{-2}$. 


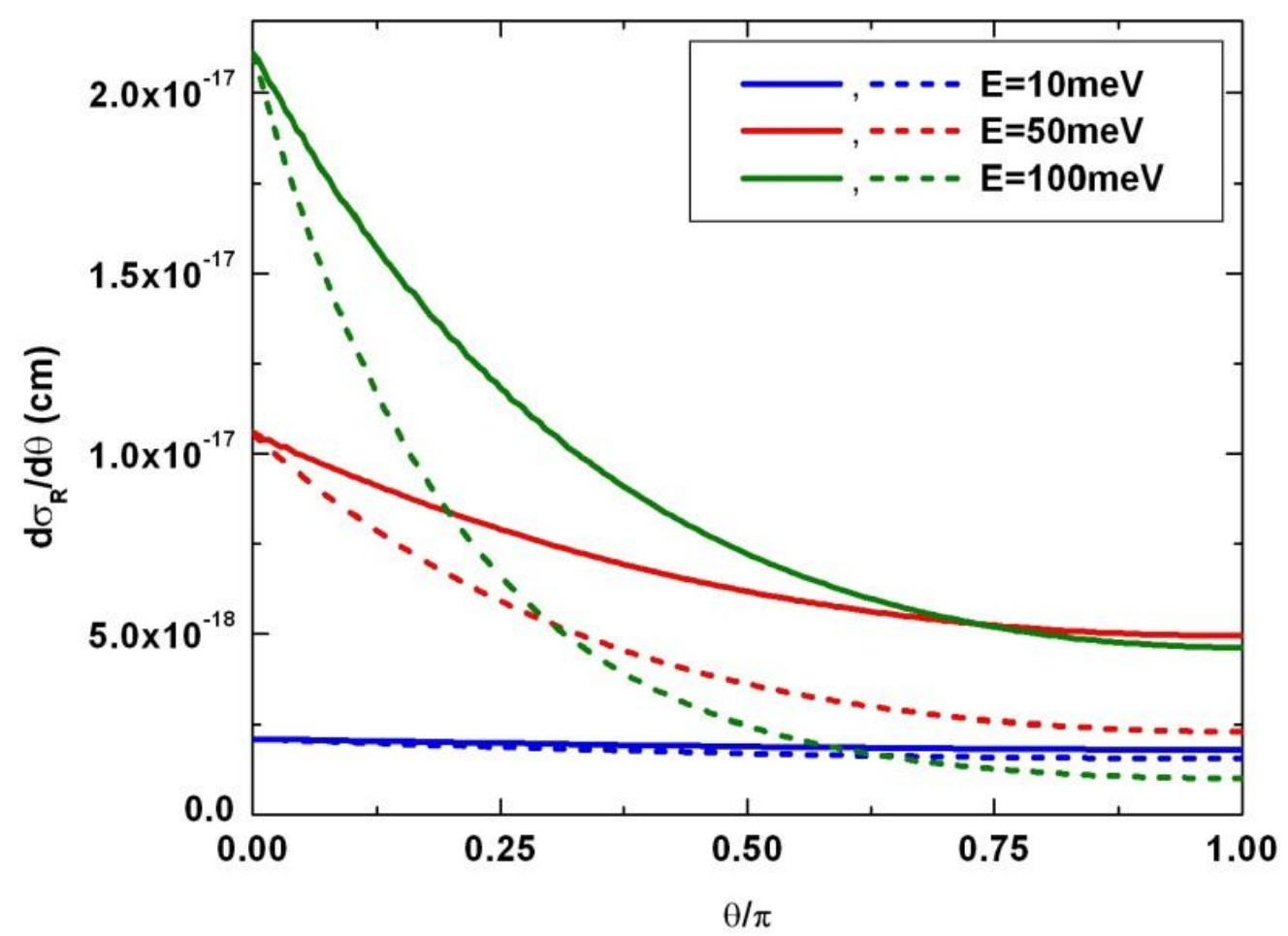

Figure 4.1: Differential scattering cross-section for spin scattering as a function of $\theta$ for several different electron energies, $z_{0}=5 \mathrm{~nm}$ (dashed lines) and $2.5 \mathrm{~nm}$ (solid lines).

Lastly, we integrate over the scattering angle to obtain the total spin-flip scattering cross-section as follows:

$\sigma_{R}=\oint\left(\frac{d \sigma_{R}}{d \theta}\right) d \theta$ 
The results as a function of electron energy for the same parameters as above are shown in figure 4.2. As interest focuses primarily on electrons near the Fermi energy, the energy scale is normalized.

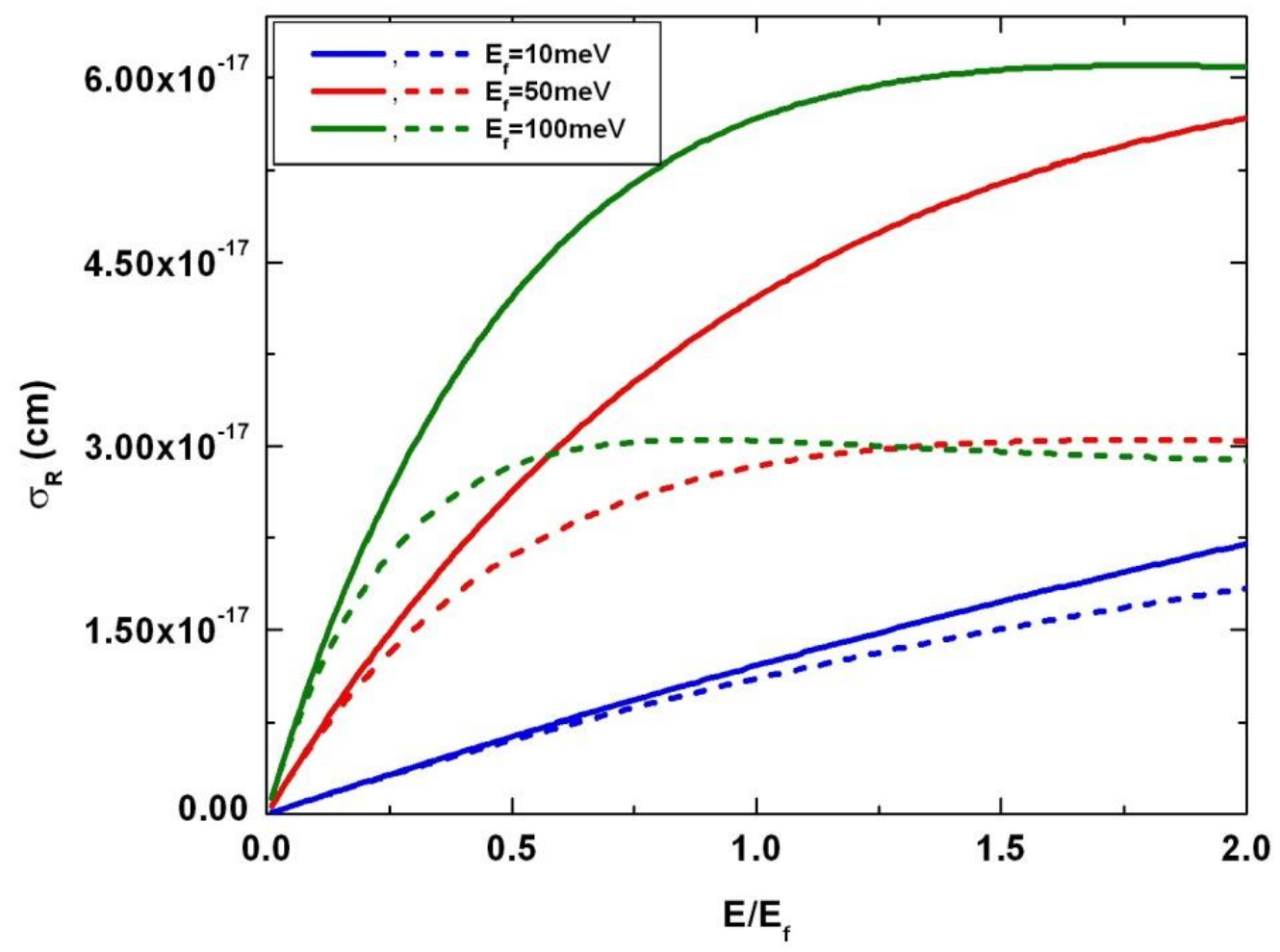

Figure 4.2: Total spin-scattering cross-section as a function of electron energy, $z_{0}=5 \mathbf{n m}$ (dashed lines) and 2.5nm (solid lines). 


\subsection{Momentum and Spin Relaxation Time}

Just as potential scattering, the Rashba mechanism can also contribute to momentum relaxation. To calculate the momentum relaxation time, the differential and total momentum cross-section are calculated as:

$\frac{d \sigma_{m, R}}{d \theta}=\frac{1}{4 \hbar^{2} v_{f}^{2}}\left(\frac{k}{8 \pi}\right)|R(q)|^{2}(1-\cos \theta)$

$\sigma_{m, R}=\oint\left(\frac{d \sigma_{m, R}}{d \theta}\right) d \theta$

For the same parameters as in section 3.4 (i.e. $N_{i}=10^{11} \mathrm{~cm}^{-2}, z_{0}=2.5 \mathrm{~nm}$ and $E_{\mathrm{f}}$ $=50 \mathrm{meV})$, the Rashba momentum relaxation time, $\tau_{\mathrm{m}, \mathrm{R}} \approx 3 \mathrm{~ms}$. This mechanism is clearly quite weak in comparison to the potential effect calculated before.

Spin relaxation however is solely associated with the Rashba term $R(q)$. By calculating the total integrated scattering cross-section as in figure 4.2, an estimate for the spin relaxation time for electrons at the Fermi energy $\left(E_{\mathrm{f}}=\right.$ $50 \mathrm{meV}$ ) yields $\tau_{\mathrm{s}} \approx 2.4 \mathrm{~ms}$ (also for the same parameters). For electrons at energies close to the Dirac point i.e. $E=E_{f}=1 \mathrm{meV}$, the spin relaxation time $\tau_{\mathrm{s}}$ is calculated to be $76 \mathrm{~ms}$ while for $E=E_{f}=100 \mathrm{meV}$, it is $1.8 \mathrm{~ms}$. 


\section{CHAPTER 5}

\section{Discussion and Conclusions}

This thesis explores extrinsic electron-scattering mechanisms in graphene. The scattering is associated with non-magnetic, charged impurities located in the substrate $\left(\mathrm{SiO}_{2}\right)$ beneath the graphene layer. Such impurities are the source of an electric field that extends through the graphene and has a non-vanishing component perpendicular to the graphene layer. The perpendicular component breaks the reflection symmetry and consequently, gives rise to a spatially varying Rashba spin-orbit interaction. This coupling mechanism may induce transitions between states of different spin. In addition to this, there is also an in-plane component of the electric field that causes conventional elastic scattering.

The graphene may contain additional charge carriers (electrons or holes) due to dopants incorporated into the graphene layer itself. Hence, the mobile charge carrier density in the graphene need not be equal to the density of charged impurities in the substrate. These mobile carriers screen the Coulomb field associated with the substrate impurity. This work primarily focuses on charge carriers incident upon the region near the impurity and experiencing a spin flip, in 
addition to the conventional screened Coulomb potential scattering event. This type of spin scattering is unique in nature as it requires a spatially varying field, and hence a spatially varying Rashba spin-orbit interaction, due to a charged center (impurity) not located within the plane of the graphene. It is quite different from the usual case of the Elliott-Yafet mechanism in which spin scattering is induced by momentum scattering between bands that are split by a spatially uniform spin-orbit interaction. In the Elliot-Yafet mechanism, essentially all the momentum relaxation scattering mechanisms can contribute to spin relaxation and therefore $\tau_{s} \propto \tau_{m}$. Here, this relationship holds only if momentum relaxation is dominated by scattering due to charged impurities in the substrate. The mechanism examined in this work is also quite different from the Dyakonov-Perel spin scattering which yields $\tau_{s} \propto\left(\tau_{m}\right)^{-1}$, because scattering dominates over the spin-orbit interaction and rapid scattering therefore effectively tends to preserve the spin orientation.

Although the calculated spin-flip scattering cross-sections for the mechanisms discussed here are small, the weakness of competing intrinsic mechanisms may lend them considerable importance in graphene spintronics. 


\section{References}

${ }^{1}$ P. R. Wallace, Phys . Rev., 71, 622 (1947)

${ }^{2}$ K.S. Novoselov, A.K. Geim, S.V. Morozov, D.Jiang, Y. Zhang, S.V. Dubonos,

I. V. Gregorieva, and A. A. Firsov, Science, 306, 666 (2004)

${ }^{3}$ Ph. Avouris, Nano Lett., 10, 4285 (2010).

${ }^{4}$ S.Cho, Y. Chen, and M.S. Fuhrer, Appl. Phys. Lett. 91, 123105 (2007).

${ }^{5}$ C. Jozsa, M. Popinciuc, N. Tombros. H.T. Jonkman, and B.J. van Wees, Phys.

Rev. Lett. 100, 236603 (2008).

${ }^{6}$ D. Huertas-Hernando, F. Guinea, and A. Brataas, Phys. Rev. B 74, 155426 (2006).

7 T. Ando, J. Phys. Soc. Jpn., 74, 777 (2005)

${ }^{8}$ T. Ando, NPG Asia Mater., 1, 17-21 (2009)

${ }^{9}$ Carbon Nanotube and Graphene Device Physics, H.-S.P. Wong, D. Akinwande (Cambridge University Press, 2011)

${ }^{10}$ A.H.C. Neto, F. Guinea, N.M.R. Peres, K.S. Novoselov, A.K. Geim, Rev. of Mod. Phys., 81, 109 (2009) 
11 H. Min, J.E. Hill, N.A. Sinitsyn, B.R. Sahu, L. Kleinman, A.H. MacDonald, Phys. Rev. B., 74, 165310 (2006)

12 C.L. Kane and E.J Mele, Phys. Rev. Lett., 95, 226801 (2005)

${ }^{13}$ D. P. DiVincenzo, E. J. Mele, Phys. Rev. B, 29, 4, 1685 (1984)

${ }^{14}$ D. S. Novikov, Phys. Rev. B, 76, 245435 (2007) 